\title{
What makes a virus a virus?
}

\section{Roland Wolkowicz and Moselio Schaechter}

In a recent review, Raoult and Forterre (Redefining viruses: lessons from mimivirus. Nature Rev. Microbiol. 6, 315-319 (2008) $)^{1}$ ) proposed a dichotomy of the biological world, dividing it into 'organisms', those entities that encode a functional translational machinery, and viruses, those entities that have capsid shells instead. The implied definition of viruses, although highly relevant, does not rely on the most fundamental aspect of what makes a virus a virus: it breaks up and loses its bodily integrity, with its progeny becoming reconstituted after replication from newly synthesized parts. We propose that the defining attribute of all viruses is their disintegration and reconstitution, from the tiny geminiviruses (15-20 $\mathrm{nm}$ diameter; $2.5 \mathrm{~kb}$ DNA genome) to the colossal Mimivirus (400 nm diameter; $800 \mathrm{~kb}$ DNA genome). Importantly, disintegration and reconstitution are totally independent of time, with reconstitution occurring minutes, days, years or centuries after disintegration.

This is true for no other cellular organism. One aspect of this cycle, the reassembly of viral constituents to make a virion, mimics many other biological assembly processes, but the dual nature of the process is what makes it unique. Capsids are essential, but they can be relegated to a list of other viral properties. This definition is pragmatic and is based not on phylogeny but on the distinctive nature of viruses. Imagine examining a particle that is surrounded by what looks like a capsid. One might suspect it to be a virus, but with no other information it could also be some other subcellular structure. If, during an infection experiment, the particle was to lose its integrity but keep its genetic information intact, and later reassemble into progeny, there would be no doubt, however, that it is a virus. We are surprised from our own experience that the world of virology has not fully embraced this outlook.

A definition of viruses that is based on their disintegration and reconstitution requires knowledge of the reproductive cycle of the biological entity to be studied. We recognize that on an operational level it is easier to examine particles under the electron microscope than it is to carry out a viral growth curve, which in fact is not achievable unless the host organism of the virus is available.

In trying to define viruses, we cannot escape a consideration of viroids and other infectious naked nucleic acids, which might have evolved from viruses that lost their capsid (thus acquiring their 'oid') or directly from nucleic acid molecules. Neither capsids nor the loss of integrity are attributes of viroids, and therefore they must be relegated to a separate category.

Roland Wolkowicz and Moselio Schaechter are at the Department of Biology and Center for Microbial Sciences, San Diego State University, San Diego, California, 92182-4614 USA. Correspondence to R.W. e-mail roland@sciences.sdsu.edu

Raoult D. \& Forterre P. Redefining viruses: lessons from mimivirus. Nature Rev. Microbiol. 6, 315-319 (2008). 